\title{
Educação em saúde: Elaboração de material educativo para síndrome mão-pé secundária ao uso da capecitabina
}

\author{
Health education: Development of educational material for hand-foot syndrome secondary to the \\ use of capecitabine \\ Educación para la salud: Desarrollo de material educativo para el síndrome mano-pie secundario \\ al uso de capecitabina
}

Fernando Césare Batista Cessel

ORCID: https://orcid.org/0000-0003-1177-9489 Universidade Estadual Paulista Júlio de Mesquita Filho, Brasil E-mail: fcessel@hotmail.com

Talita Oliveira de Lima

ORCID: https://orcid.org/0000-0002-6617-65149 Hospital das Clínicas da Faculdade de Medicina de Botucatu, Brasil E-mail: talima@gmail.com

Mayara Spin

ORCID: https://orcid.org/0000-0003-4545-608X Universidade Estadual Paulista Júlio de Mesquita Filho, Brasil E-mail: mayara.spin@unesp.br

Tânia dos Santos Salvador

ORCID: https://orcid.org/0000-0002-1697-3603 Hospital das Clínicas da Faculdade de Medicina de Botucatu, Brasil E-mail: taniadossantossalvador@gmail.com

Bruna Santos Ezequiel ORCID: https://orcid.org/0000-0002-3127-1634 Universidade Estadual Paulista Júlio de Mesquita Filho, Brasil E-mail: bru.ezequiel@hotmail.com

Karina Alexandra Batista da Silva Freitas ORCID: https://orcid.org/0000-0002-3413-8468 Hospital das Clínicas da Faculdade de Medicina de Botucatu, Brasil E-mail: k.freitas@unesp.br

\begin{abstract}
Resumo
O câncer é o principal problema de saúde pública no mundo. Os fármacos antineoplásicos podem apresentar graves reações adversas, muitas delas são potencialmente fatais se não forem manejadas adequadamente. A capecitabina é um pró-fármaco quimioterápico sendo frequentemente utilizada no tratamento do câncer de mama, cólon e reto. Dentre as toxicidades mais frequentes em pacientes submetidos ao uso de capecitabina está a síndrome mão-pé (SMP). O objetivo deste trabalho foi elaborar um folder de orientações para prevenção e tratamento da SMP direcionado aos pacientes que usam a capecitabina visando à otimização do seu tratamento. Trata-se de relato de experiência que envolveu três etapas: identificação do problema; revisão narrativa da literatura, desenvolvimento do layout, ilustrações e escrita em formato de folder. O material constou de orientações simples, demonstrando quais os principais sinais e sintomas da SMP, além de um telefone para contato com o serviço. Foram inseridas também, questões relacionadas a mudança de hábitos de vida como melhora da ingestão hídrica, uso de sapatos confortáveis e a temperatura da água do banho, além de cuidados com a manipulação de produtos de limpeza. Além disso, foram citados os métodos não farmacológicos como uso de cremes à base de uréia, aloe vera e laserterapia. Concluímos que a elaboração do folder propôs um material educativo impresso que possibilitou abordagem mais assertiva, direcionada ao indivíduo, esclarecendo suas dúvidas e assegurando um melhor tratamento.
\end{abstract}

Palavras-chave: Neoplasia; Antineoplásicos; Capecitabina; Síndrome mão-pé; Educação em saúde.

\begin{abstract}
Cancer is the main public health problem in the world. Anticancer drugs can have serious adverse reactions, many of which are potentially fatal if not properly managed. Capecitabine is a chemotherapeutic prodrug that is frequently used in the treatment of breast, colon and rectal cancer. Among the most frequent toxicities in patients undergoing capecitabine use is hand-foot syndrome (HFS). The objective this study was to develop a folder of guidelines for the prevention and treatment of HFS aimed at patients who use capecitabine in order to optimize their treatment. This is an experience report that involved three steps: problem identification; narrative literature review, layout development,
\end{abstract}


illustrations and writing in folder format. The material consisted of simple guidelines, demonstrating the main signs and symptoms of HFS, in addition to a telephone number for contacting the service. Questions related to changing lifestyle habits were also included, such as improving water intake, wearing comfortable shoes and the temperature of the bath water, as well as care with the handling of cleaning products. In addition, non-pharmacological methods were mentioned, such as the use of urea-based creams, aloe vera and laser therapy. We concluded that the elaboration of the folder proposed a printed educational material that allowed a more assertive approach, aimed at the individual, clarifying their doubts and ensuring better treatment.

Keywords: Neoplasm; Antineoplastics; Capecitabine; Hand-foot syndrome; Health education.

\section{Resumen}

El cáncer es el principal problema de salud pública del mundo. Los medicamentos contra el cáncer pueden tener reacciones adversas graves, muchas de las cuales son potencialmente fatales si no se manejan adecuadamente. La capecitabina es un profármaco quimioterapéutico que se utiliza con frecuencia en el tratamiento del cáncer de mama, colon y recto. Entre las toxicidades más frecuentes en pacientes sometidos a uso de capecitabina se encuentra el síndrome mano-pie (SMP). El objetivo de este estudio fue desarrollar una carpeta de guías para la prevención y el tratamiento del SPM dirigida a pacientes que usan capecitabina con el fin de optimizar su tratamiento. Este es un informe de experiencia que involucró tres pasos: identificación del problema; revisión de literatura narrativa, desarrollo de maquetación, ilustraciones y redacción en formato de carpeta. El material constaba de pautas sencillas, que demostraban los principales signos y síntomas del SMP, además de un número de teléfono para contactar con el servicio. También se incluyeron cuestiones relacionadas con el cambio de hábitos de vida, como la mejora de la ingesta de agua, el uso de zapatos cómodos y la temperatura del agua del baño, así como el cuidado con el manejo de los productos de limpieza. Además, se mencionaron métodos no farmacológicos, como el uso de cremas a base de urea, aloe vera y terapia con láser. Concluimos que la elaboración de la carpeta proponía un material educativo impreso que permitía un abordaje más asertivo, dirigido al individuo, aclarando sus dudas y asegurando un mejor trato.

Palabras clave: Neoplasma; Antineoplásticos; Capecitabina; Síndrome mano-pie; Educación para la salud.

\section{Introdução}

O câncer é o principal problema de saúde pública no mundo e já está entre as quatro principais causas de morte prematura (antes dos 70 anos de idade) na maioria dos países. A incidência e a mortalidade vêm aumentando em parte pelo envelhecimento e crescimento populacional, como também pela mudança na distribuição e na prevalência dos fatores de risco para neoplasia, especialmente os associados ao desenvolvimento socioeconômico. Para o Brasil, a estimativa para cada ano do triênio 2020-2022 é de 625 mil casos novos. O câncer de pele não melanoma será o mais incidente, seguido pelos cânceres de mama, próstata, cólon e reto, pulmão e estômago (Bray et al., 2018; Instituto Nacional do Câncer, 2019; Mathers et al., 2003).

A quimioterapia antineoplásica (QTA) é um dos principais tratamentos utilizados no combate ao câncer. Desta forma, aumenta-se o risco de interações medicamentosas e reações adversas a medicamento (RAM), promovendo alteração no estado físico e por vezes mental decorrente dos efeitos colaterais e pela agressividade da própria doença (Martins et al., 2019; Medina et al., 2013).

As RAM são definidas como qualquer resposta prejudicial ou indesejável, não intencional, a um medicamento, que se manifesta após a administração de doses recomendadas para profilaxia, diagnóstico ou tratamento farmacoterapêutico de doenças, podendo determinar mudanças na conduta clínica traçada pelo profissional da área de saúde. Considera-se que tais ocorrências são causas expressivas de hospitalização, de aumento do tempo de permanência hospitalar e até mesmo de óbito (Martins et al., 2019; Modesto et al., 2016).

A QTA está associada ao desenvolvimento de RAM graves, as quais quando não manejadas de forma correta e em tempo hábil, podem ser fatais. Cabe ao farmacêutico possuir conhecimentos sobre estas reações a fim de orientar e sanar as dúvidas dos pacientes em tratamento (Bertella et al., 2020; Martins et al., 2019).

A capecitabina é uma QTA de administração por via oral, comumente utilizada no tratamento do câncer de mama, cólon e reto. Trata-se de um carbamato de fluoropirimidina altamente absorvida no trato gastrointestinal. O metabolismo ocorre no fígado e no tecido tumoral, onde ocorre a conversão da droga em 5-FU, obtendo uma disponibilidade trinta vezes 
maior do que no plasma. Possui uma meia-vida entre 0,7 e 1,14 horas, cuja via de eliminação é majoritariamente renal (Fernandes et al., 2019; Martins et al., 2019; Xeloda, 2020).

As RAM mais frequentes em pacientes são: diarréia, estomatite, náusea, vômito, alopécia, eritrodisestesia palmoplantar também conhecida como síndrome mão-pé (SMP) e neutropenia. Estudos comprovam que mais de 50\% dos pacientes tratados com o fármaco, desenvolvem a SMP, acarretando grande impacto na sua qualidade de vida (Fernandes et al., 2019; Simão et al., 2012). O desenvolvimento da SMP pode ser responsável pela redução da dose do medicamento ou até mesmo a suspensão do tratamento do câncer (Costa et al., 2019; Júnior et al., 2017).

Essa síndrome é desenvolvida pelo aumento de timidina fosforilase nos queratinócitos da pele e a capecitabina afeta o sistema glandular écrino levando a um acúmulo de metabólitos de capecitabina (Fernandes et al., 2019).

Desta forma, com a exposição das mãos e pés ao calor, bem como o atrito entre as palmas das mãos e plantas dos pés, causando a dilatação dos vasos capilares provocando eritema semelhante a uma queimadura. As áreas afetadas podem se tornar secas e descascar, apresentando dormência e parestesia. Se não for adequadamente tratada, pode evoluir para uma condição muito dolorosa e debilitante, causando grande desconforto e levando a uma piora na qualidade de vida do paciente em tratamento com o fármaco (Sanches, 2017).

O National Câncer Institute (NCI) dos Estados Unidos, elaborou o Commom Terminology Criteria for Adverse Events (CTCAE 5,0) que classifica os eventos adversos em diferentes graus, classificando a SMP da seguinte forma (NCI, 2017):

- $\quad$ Estágio I: Alterações cutâneas mínimas ou dermatite (por exemplo, eritema, edema ou hiperqueratose) sem dor;

- $\quad$ Estágio II: Alterações da pele (por exemplo, descamação, bolhas, sangramento, fissuras, edema ou hiperceratose) com dor e limitação de atividades diária;

- Estágio III: Alterações graves da pele (por exemplo, descamação, bolhas, sangramento, fissuras, edema ou hiperceratose) com dor e limitação diária de autocuidado. Casos mais graves podem levar a infecções locais e generalizadas, causando risco à vida do paciente (Costa et al., 2019; Fernandes et al., 2019; Sanches, 2017).

Neste contexto, o objetivo deste trabalho foi elaborar um folder de orientações para prevenção e tratamento da SMP direcionado aos pacientes que usam a capecitabina visando a otimização do seu tratamento.

\section{Metodologia}

Trata-se de um relato de experiência sobre a confecção de um folder de orientação para os pacientes que usam a capecitabina na prevenção e tratamento da SMP.

Para a construção do folder foi realizado um estudo metodológico, que trata do desenvolvimento, da validação e da avaliação de ferramentas e métodos de pesquisa (Polit \& Beck, 2011). O estudo foi realizado em três etapas: a) elaboração da revisão narrativa para a construção do corpo de conhecimento a cerca da etiologia, fatores de risco, prevenção, sinais e sintomas e tratamento da SMP. A pesquisa foi realizada no período de novembro de 2020 a maio de 2021 no Pubmed, Scielo, Google Scholar e sites como INCA - Instituto Nacional de Câncer e Sobrafo - Sociedade Brasileira de Farmacêuticos em Oncologia. Os critérios de inclusão foram artigos que retratassem questões relacionadas aos sintomas, maneiras de prevenção e tratamento da SMP, com textos completos disponíveis, na língua inglesa e portuguesa.

b) Confecção do folder de forma didática, com o uso de ilustrações e textos de linguagem simples e de fácil entendimento. A formatação e diagramação foi realizada utilizando-se o programa Canva ${ }^{\circledR}$. 
c) Após a elaboração, o folder foi enviado a juízes (enfermeiros e farmacêuticos com experiência em oncologia) e está em processo de revisão e validação para posteriormente ser incorporado às rotinas da farmácia do Ambulatório de Oncologia do Hospital Estadual de Botucatu.

\section{Resultados e Discussão}

Com a revisão narrativa identificou-se estudos que abordavam tratamentos farmacológicos e não-farmacológicos para pacientes acometidos pela SMP. Também foram encontradas referências aos mecanismos de prevenção e mudanças nos hábitos de vida.

\section{Tratamentos farmacológicos}

A reposição de vitamina B6 (piridoxina) é um fator de proteção para a SMP, uma vez que este composto quando deficiente pode promover o surgimento de dermatites, ocasionando casos graves desta síndrome. Em contrapartida, pacientes com doses ideais de vitamina B6 podem apresentar um fator de proteção ao desenvolvimento da síndrome (Pereira et al., 2020).

Os analgésicos da classe dos anti-inflamatórios não esteroides são bastante utilizados na prática clínica. A sua ação analgésica se relaciona com a inibição de prostaglandinas, substâncias que sensibilizam nociceptores cujo bloqueio promove analgesia. Para os casos mais graves, foram utilizados anti-inflamatórios esteroides da classe dos glicocorticoides (prednisolona, mometasona, dexametasona e triancinolona). Esses agentes terapêuticos apresentam um grande potencial antiinflamatório (Pereira et al., 2020).

O uso de anti-inflamatórios não esteróides (AINES) tem eficácia comprovada na prevenção e no tratamento da SMP, por reduzirem a inflamação. A administração concomitante da capecitabina e celecoxibe, inibidor seletivo da COX-2, pode prevenir a ocorrência da SMP. Um estudo de meta-análise que avaliou medidas preventivas contra a SMP como o uso de celecoxibe, piridoxina e uréia tópica, demonstrou que o celecoxibe promoveu uma redução significante do risco em casos de SMP graus 2 ou 3 em cerca de 50\% dos casos (Macedo et al., 2014; Zhang et al., 2012).

Entretanto, são necessários dados maiores de estudos duplo-cegos para confirmar sua eficácia. Além disso, os potenciais efeitos colaterais, incluindo riscos cardiovasculares e sangramento gastrointestinal superior, devem ser levados em consideração (Chen et al., 2008; Johnsen, 2005; Macedo et al., 2014).

\section{Tratamentos não farmacológicos}

Em uma revisão sistemática com meta-análise, observou-se que os sinais e sintomas da SMP devem ser detectados precocemente, e a partir desse momento medidas de suporte devem ser iniciadas como: uso de meias de algodão, sapatos macios a fim de evitar pontos de pressão, evitar extremos de temperatura e fricção de pele. Alguns tratamentos não farmacológicos indicados, incluem agentes tópicos antes e depois do aparecimento dos sintomas, no sentido de manter a hidratação da pele e garantir sua integridade. Vários agentes podem ser utilizados, como aloe vera, sulfato de magnésio, pomada a base de lanolina e uréia (Watkins et al., 2014). Evidencia ainda, que a escolha dos agentes tópicos deve ser realizada de acordo com a classificação da SMP, variando desde cremes de uréia, ácido acetilsalicílico para os eventos estágio I, corticoesteróides e anestésicos tópicos para estágio II até o uso de opióides, agonistas GABA e lidocaína 2\% para eventos estágio III ( $\mathrm{Li} \& \mathrm{Gu}, 2017)$.

O primeiro tópico abordado no folder como forma de prevenção é o uso da crioterapia aplicada por meio de compressas com gelo. Ao realizar a compressa ocorre a estimulação da vasoconstrição local, reduz o edema, a dor e tem ação anti-inflamatória (Pereira et al., 2020; Li \& Gu, 2017). 
A hidroterapia é uma das estratégias não-farmacológicas que apresentou bons resultados frente a esta síndrome. Dentre os seus benefícios, foi identificado ações de: hidratação, emoliência e anti-inflamatória (Pereira et al., 2020). Os cremes hidratantes a base de aloe vera (babosa) possuem propriedades umectantes, emolientes, anti-inflamatórias e regeneradores de tecido. Estudos mostram que a manose-6-fosfato, polissacarídeo presente neste composto ativo pode apresentar capacidade cicatrizante, por meio do estímulo das atividades dos macrófagos e fibroblastos, aumentando a síntese de colágeno e proteoglicanas, promovendo a reparação de tecidos (Hekmatpou et al., 2019; Richardson et al., 2005; Simão et al., 2012).

De acordo com a cartilha elaborada pelo Instituto Viver, existem outras opções de cremes hidratantes e umectantes para serem utilizados na prevenção e tratamento dos sinais e sintomas, dentre eles: a alantoína que possui propriedades hidratantes, queratolíticas, descamantes de pele, suavizadoras, protetoras contra agentes irritantes e curativas; Epiderfill que é um ácido hialurônico microencapsulado que causa hidratação imediata; Hidroviton, uma mistura de aminoácidos, lactato sódico, uréia e alantoína; Óleo vegetal puro que lubrifica e regenera a pele; vitaminas A, C e E com ação regeneradora, reparadora, anti-oxidante, hidratante, melhorando a imunidade, firmeza e elasticidade da pele e o Ômega, potente fotoprotetor e anti-inflamatório na pele (Sanches, 2017).

Além das possibilidades de cremes hidratantes já descritas, tem-se como prescrição recomendada rotineiramente pela equipe de oncologia do nosso serviço, o hidratante a base de uréia 10\%. A uréia aumenta efetivamente a capacidade da pele em absorver e reter a umidade e age como um queratolítico suave, promovendo uma barreira epidérmica protetora contra agressores que promoveriam o ressecamento da pele (Thalita, 2017).

Outra opção que pode ser utilizada na SMP é a fotobiomodulação (FBM), também conhecida como laserterapia de baixa intensidade (LBI). A utilização da FBM auxilia na regeneração dos tecidos (pele), tem ação analgésica, anti-inflamatória, cicatrizante e normalizadora, devolvendo à pele o seu estado original. O princípio da FBM é a irradiação celular utilizando-se radiação não ionizante para modificar a atividade celular (Rezende \& Lenzi, 2020).

Sendo assim, existe um consenso quanto a utilização dos comprimentos de ondas a serem utilizados, sendo que os comprimentos entre 600 e 700nm são preferíveis frente ao tratamento de tecidos superficiais e os comprimentos de 780 e 950nm, para tecidos de localização mais profunda. A luz é absorvida pelas mitocôndrias e pela membrana celular. Para tanto, é necessário a presença de cromóforos que são fotoreceptores. O principal cromóforo ativado pelo laser vermelho é o citocromo C oxidase (CCO) (Lins et al., 2010; Trivelin et al., 2020).

Durante um processo de desequilíbrio no organismo (processo inflamatório, lesões, efeitos colaterais), ocorre um processo secundário no qual as mitocôndrias produzem óxido nítrico (NO), que se ligam aos CCO, movendo o oxigênio e inibindo a respiração celular. Com isso ocorre a diminuição da adenosina trifosfato (ATP), aumentando o estresse oxidativo. A utilização da luz vermelha nesta etapa promove um fotodesligamento do NO e o oxigênio volta a ligar-se no CCO, diminuindo o estresse oxidativo e como consequência, ocorre um aumento das espécies reativas de oxigênio (ROS), ATP e íons de Ca ${ }^{2+}$. Esse processo leva a diferenciação, proliferação e migração celular (Freitas, 2020; Phaniendra et al., 2014).

Pela FBM apresentar um feito reparador, é possível prevenir e tratar a SMP utilizando-se o comprimento de onda vermelho (entre 600 e 700nm. O uso da FBM duas vezes por semana, por quatro semanas, apresenta resultados satisfatórios (Rezende \& Lenzi, 2020).

Já é comprovado que a FBM é empregada com grandes respostas em pacientes oncológicos para prevenção e tratamento das mucosites e radiodermites e para prevenção da lesão de pele em extravasamentos de antineoplásicos, por diminuir o processo inflamatório e dor. Visto isso, pelo mesmo propósito, é possível a utilização na SMP (Freitas et al, 2020; Robijns et al., 2017; Zecha et al., 2016). 
Mesmo representando uma abordagem útil para a SMP, faz-se necessário estudos multicêntricos, assim como a importância de reconhecer os primeiros sinais e sintomas para implementar intervenções de FBM individualizadas (Latifyan et al., 2019).

A FBM já é empregada no ambulatório de oncologia do Hospital Estadual Botucatu para tratar e prevenir diversos tipos de lesões de pele, bem como eventos adversos relacionados à terapia antineoplásica. Após a revisão de literatura realizada para a confecção desse folder, esse procedimento foi incorporado na prevenção e tratamento de SMP.

\section{Mudanças nos hábitos de vida}

Dentre os principais hábitos de vida que são recomendados para a prevenção para SMP estão medidas que evitem a pressão, fricção e dilatação de vasos sanguíneos, como o exemplo de: uso de banhos quentes, exposição ao sol, uso de roupas de algodão e sapatos confortáveis (Sanches, 2017; Xeloda, 2020).

O ressecamento da pele é comum em pacientes em uso de quimioterápicos, sendo assim, é imprescindível manter os níveis de hidratação do organismo, sendo ele de forma tópica, ou por meio do aumento da ingesta hídrica, evitando o ressecamento que causa o rompimento da barreira epidérmica e redução dos níveis de água do organismo, aumentando o risco de lesões. De encontro a isso, podemos citar a transpiração excessiva, sendo recomendado o uso de roupas leves, evitando a perda de água por meio da sudorese excessiva (Addor \& Aoki, 2010; Ozkol et al., 2013; Richards et al., 2012; Simão et al., 2012).

Outros pontos importantes levantados acerca de mudança nos hábitos de vida, visando a prevenção da SMP foi a inserção do uso de luvas de borracha para manipular os produtos de limpeza, evitando o contato da pele com produtos químicos e irritantes e o uso de meias de algodão para dormir, de modo com que estas aumentam a absorção dos cremes hidratantes (Sanches, 2017).

Desta forma, mediante todas as informações encontradas na revisão, elaborou-se o folder com objetivo de promover orientação ao paciente. O material constou de orientações simples, demostrando quais os principais sinais e sintomas da SMP, além de um telefone para contato com o serviço. Foram inseridos também, questões relacionadas a mudança de hábitos de vida como melhora da ingestão hídrica, uso de sapatos confortáveis e a temperatura da água do banho, além de cuidados com a manipulação de produtos de limpeza. Além disso, foram citados os métodos não farmacológicos como uso de cremes a base de uréia, aloe vera, laserterapia. Em relação as questões farmacológicas, optou-se por não colocar no folder para evitar o uso de medicamentos sem prescrição médica. 
Research, Society and Development, v. 10, n. 17, e114101724093, 2021

(CC BY 4.0) | ISSN 2525-3409 | DOI: http://dx.doi.org/10.33448/rsd-v10i17.24093

Figura 1 - Folder de orientações para prevenção e tratamento da SMP direcionado aos pacientes que usam a capecitabina (em processo de validação). Botucatu, São Paulo, Brasil, 2021.
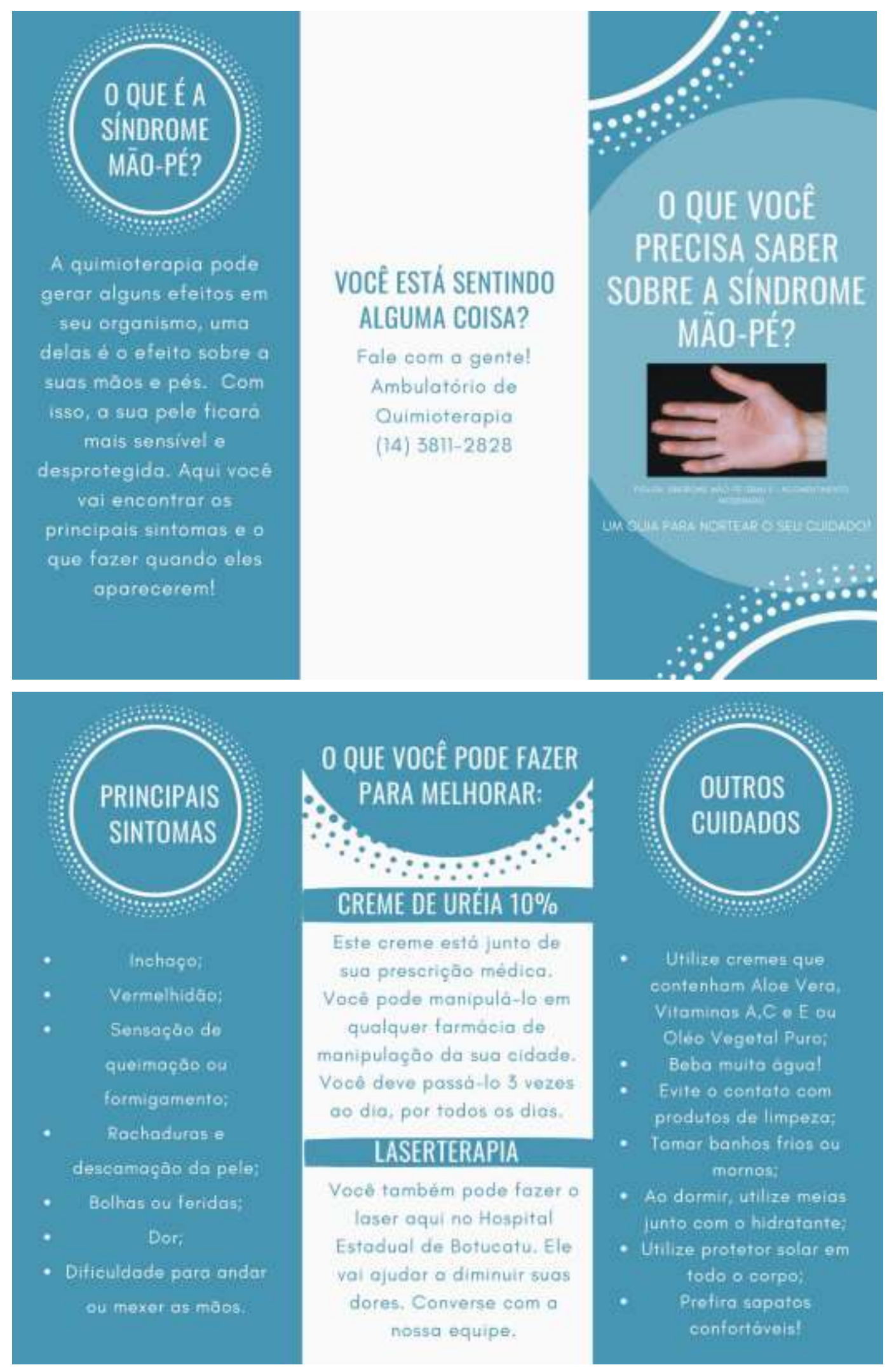

Fonte: Autores (2021). 


\section{Conclusão}

Visando proporcionar a otimização do tratamento com o objetivo de reduzir o adiamento e/ou suspensão do tratamento, redução de dose e prevenção de complicações, a elaboração do folder propôs um material educativo impresso que possibilitará abordagem mais assertiva, direcionada ao indivíduo, esclarecendo suas dúvidas e assegurando um melhor tratamento.

Sugerimos que futuramente, após a validação do material, mais pesquisas sejam realizadas com os resultados de acompanhamento desses pacientes.

\section{Referências}

Addor F. A. S., \& Aoki V. (2010). Barreira cutânea na dermatite atópica. Anais Brasileiros de Dermatologia, 85 (2), $184-94$.

Bertella T., Pretto L. C., Pasinato R., Picinin D., Ebel A., Schorn G. D., \& Mioso C. (2020). Acompanhamento farmacoterapêutico na dispensação de medicamentos antineoplásicos via oral: relato de experiência. I Simpósio Sul Brasileiro de Oncologia Clínica e Cirúrgica, v 1, n 1.

Bray F., Ferlay J., Soerjomataram I., Siegel R. L., Torre L. A., \& Jemal A. (2018). Global cancer statistics 2018: GLOBOCAN estimates of incidence and mortality worldwide for 36 cancers in 185 countries. CA: A Cancer Journal for Clinicians, 68 (6), 394-424.

Chen, Y., Jobanputra, P., Barton, P., Bryan, S., Fry-Smith, A., Harris, G., \& Taylor, R. (2008). Cyclooxygenase-2 selective non-steroidal anti-inflammatory drugs (etodolac, meloxicam, celecoxib, rofecoxib, etoricoxib, valdecoxib and lumiracoxib) for osteoarthritis and rheumatoid arthritis: a systematic review and economic evaluation. Health Technology Assessment, 12 (11).

Costa J. S., Silva G. M., Kameo S. Y., Amorim B. F., \& Ramos M. J. O. (2019). Síndrome mão-pé induzida por quimioterapia: abordagem clínica e epidemiológica de pacientes com câncer. Rev Bras Cancerol, 65 (2).

Fernandes C. D. L., Castro C.Q., Franciele E., Maria V., \& Bayer L. (2019). Vigilância em Saúde: ações de promoção, prevenção, diagnóstico e tratamento. Perfis dos pacientes em tratamento quimioterápico com capecitabina no hospital universitário de Santa Maria/RS. $6^{\circ}$ Congresso Internacional em Saúde.

Freitas, K. A. B. S. (2020). Efeitos da fotobiomodulação no extravasamento de antraciclina: estudo experimental. Universidade Estadual Paulista "Júlio de Mesquita Filho", Faculdade de Medicina de Botucatu.

Freitas, K. A. B. S., Lima, T. O., Minicucci, E. M., Silva, K. A. B., \& Vigliassi, A. P. (2020). Association of photobiomodulation and topical hyaluronidase in extravasation and infiltration of antineoplastics. Retrospective stud. Revista Nursing, 23 (271), 4975-4978.

Hekmatpou D., Mehrabi F., Rahzani K., \& Aminiyan A. (2019). The Effect of Aloe Vera Clinical Trials on Prevention and Healing of Skin Wound: A Systematic Review. Iranian Journal of Medical Sciences, 44 (1), 1-9.

Johnsen S. P. (2005). Risk of Hospitalization for Myocardial Infarction Among Users of Rofecoxib, Celecoxib, and Other NSAIDs. Archives of Internal Medicine, 165 (9), 978.

Júnior W. B., Tomaz A. C. A., Vieira L. F. A., Silva P. M. X., Oliveira S. M. B., \& Lima M. K. S. (2017). Capecitabine induced hand-foot syndrome-a case report. Cogitare Enferm, 22 (1), 01-04.

Latifyan S., Genot M. T., Fernez B., Scharll M. F., \& Klastersky J. A. (2019). Use of low-level laser therapy (LLLT) or photobiomodulation (PBM) for the management of the hand-foot syndrome (HSF) or palmo-plantar erythrodysesthesia (PPED) associated with cancer therapy. Supportive Care in Cancer, 28 (7), 3287-90.

Li J., \& Gu J. (2017). Hand-foot skin reaction with vascular endothelial growth factor receptor tyrosine kinase inhibitors in cancer patients: A systematic review and meta-analysis. Critical Reviews in Oncology/Hematology, 119, 50-8.

Lins R. D. A. U., Dantas E. M., Lucena K. C. R., Catão M. H. C. V., Granville-Garcia A.F., \& Carvalho Neto L.G. (2010). Efeitos bioestimulantes do laser de baixa potência no processo de reparo. Anais Brasileiros de Dermatologia, 85 (6), 849-55.

Macedo L. T., Lima J. P., Santos L. V., \& Sasse A. D. (2014) Prevention strategies for chemotherapy-induced hand-foot syndrome: a systematic review and meta-analysis of prospective randomized trials. Support Care Cancer. 22 (6), 1585-93.

Martins T. L., Pinto A. R., Pires J. M. D., Silva M. J. S. D., Couto D. H. N., \& Elias S.C. (2019). Adverse reaction induced by capecitabine: the importance of pharmacovigilance | Revista Brasileira de Farmácia Hospitalar e Serviços de Saúde. Revista Brasileira de Farmácia Hospitalar e Serviços de Saúde. http://www.rbfhss.org.br/sbrafh/article/view/163

Mathers, C. D., Bernard, C., Iburg, K. M., Inoue, M., Ma Fat, D., Shibuya, K., Stein, C., Tomijima, N., \& Xu, H. (2003). Global Burden of Disease in 2002: data sources, methods and results. World Health Organization. 54th ed.

Medina A. M., Leal A. F., Zavaglia G. O., Muniz R. M., Guimarães S. R. L., \& Faes A. D. R. (2013). A consulta de enfermagem como estratégia de cuidado ao cliente oncológico em tratamento radioterápico. Ciência, Cuidado e Saúde, 7. https://doi.org/10.4025/cienccuidsaude.v7i0.6727.

Modesto A. C. F., Ferreira T. X. A. M., Provin M. P., Amaral R. G., \& Lima D. M. (2016). Reações Adversas a Medicamentos e Farmacovigilância: Conhecimentos e Condutas de Profissionais de Saúde de um Hospital da Rede Sentinela. Revista Brasileira de Educação Médica, 40 (3), $401-10$. 
National Cancer Institute (US). Division of cancer treatment and diagnosis. Commom toxicity criteria (Computer Program). Version 5.0 NCI; 2017.

Ozkol, H. U., Calka, O., \& Bulut, G. (2013). Chemotherapy-induced acral erythema with involvement of the face and neck. Toxicology and Industrial Health, $32(5), 791-4$

Pereira, P. P., Pedroso, R. S., \& Ribeiro, M. A. (2020). Identificação, Prevenção e Tratamento da Síndrome Mão-Pé Induzida por Quimioterapia: Revisão Sistemática. Revista Brasileira de Cancerologia, 65 (4).

Phaniendra, A., Jestadi, D. B., \& Periyasamy L. (2014). Free Radicals: Properties, Sources, Targets, and Their Implication in Various Diseases. Indian Journal of Clinical Biochemistry, 30 (1), 11-26.

Polit, D. F. \& Beck, C. T. (2011). Fundamentos de pesquisa em enfermagem: avaliação de evidências para a prática da enfermagem. Porto Alegre: Artmed, $7^{\text {a }}$ ed.

Rezende, R., \& Lenzi, J. (2020). Eletrotermofooterapia em Oncologia: da evidência à prática clínica. 1. ed. Rio de Janeiro: Thieme Revinter Publicações, 312 p.

Richardson, J., Smith J. E., McIntyre M., Thomas, R., \& Pilkington, K. (2005). Aloe Vera for Preventing Radiation-induced Skin Reactions: A Systematic Literature Review. Clinical Oncology, 17 (6), 478-84.

Richards, K. N., Ivan, D., Rashid, R. M., \& Chon, S. Y. (2012). Paclitaxel-Induced Acral Erythema. Archives of Dermatology, 148 (11), 1333.

Robijns, J., Censabella, S., Claes, S., Pannekoeke, L., Bussé, L., Colson, D., Kaminski, I., Lodewijckx, J., Bulens, P., Maes, A., Noé, L., Brosens, M., Sanches O. (2017). Síndrome Mão-Pé: prevenção, diagnóstico e tratamento. [Cartilha]. Instituo Viver Hoje. www.viverhoje.org.

Simão, D. A. S., Lima, E. D. R. P., Souza, R. S., Faria, T. V., \& Azevedo, G. F. (2012). Síndrome mão-pé induzida por quimioterapia: relato de um caso. Revista Brasileira de Enfermagem, 65 (2), 374-8.

Thalita, M. (2017). Ureia para prevenção da eritrodisestesia palmo-plantar em pacientes submetidos à quimioterapia: revisão sistemática. https://bdm.unb.br/handle/10483/18411.

Trivelin, M. L. O. A. A., Alchorne, A. O. A., Marques, E. R. M. C., Alchorne, M. M. A., Silva, M. F., Freixadas, E. M. R., Trivelin, S. A. A., \& Chavantes, M. C. (2020). Avaliação do efeito clínico da terapia de fotobiomodulação com uso do laser de baixa intensidade em rosácea eritêmato-telangiectásica: Estudo piloto. Brazilian Journal of Development, 6 (7), 50152-50175.

Watkins, A. A., Johnson, T. V., Shrewsberry, A. B., Nourparvar, P., Madni, T., Watkins, C. J., Feingold, P. L., Kooby, D. A., Maithel, S. K., Staley, C. A., \& Master, V. A. (2014). Ice Packs Reduce Postoperative Midline Incision Pain and Narcotic Use: A Randomized Controlled Trial. Journal of the American College of Surgeons, 219 (3), 511-517.

Xeloda (capecitabina). [Bula]. Rio de Janeiro: Produtos Roche Químicos e Farmacêuticos S.A; 2020.

Zhang, R. X., Wu, X. J., Wan, D. S., Lu, Z. H., Kong, L. H., Pan, Z. Z., \& Chen, G. (2012). Celecoxib can prevent capecitabine-related hand-foot syndrome in stage II and III colorectal cancer patients: result of a single-center, prospective randomized phase III trial. Annals of Oncology, 23(5), 1348-1353.

Zecha, J. A. E. M., Raber-Durlacher, J. E., Nair, R. G., Epstein, J. B., Sonis, S. T., Elad, S., Hamblin, M. R., Barasch, A., Migliorati, C. A., Milstein, D. M. J., Genot, M.-T., Lansaat, L., van der Brink, R., Arnabat-Dominguez, J., van der Molen, L., Jacobi, I., van Diessen, J., de Lange, J., Smeele, L. E., Bensadoun, R.-J. (2016). Low level laser therapy/photobiomodulation in the management of side effects of chemoradiation therapy in head and neck cancer: part 1: mechanisms of action, dosimetric, and safety considerations. Supportive Care in Cancer, 24 (6), 2781-2792. 